\title{
Eddy Current Scanning of Niobium for SRF Cavities at Fermilab
}

\author{
C. Boffo, P. Bauer, M. Foley, C. Antoine, C. Cooper and A. Brinkmann
}

\begin{abstract}
In the framework of SRF cavity development, Fermilab is creating the infrastructure needed for the characterization of the material used in the cavity fabrication. An important step in the characterization of "as received" niobium sheets is eddy current scanning. Eddy current scanning is a nondestructive technique first adopted and further developed by DESY with the purpose of checking the cavity material for subsurface defects and inclusions.

Fermilab has received and further upgraded a commercial eddy current scanner previously used for the SNS project. This scanner is now used daily to scan the niobium sheets for the Fermilab third harmonic, the ILC, and the Proton Driver cavities. After optical inspection, more than 400 squares and disks have been scanned and when necessary checked at the optical and electron microscopes, anodized, or measured with profilometers looking for surface imperfections that might limit the performance of the cavities.
\end{abstract}

This paper gives a status report on the scanning results obtained so far, including a discussion of the classification of signals being detected.

Index Terms-SRF cavities, ILC, Niobium, Eddy Current Scanning.

\section{INTRODUCTION}

$\mathrm{T}$ he use of Eddy Current Scanners (ECS) in SRF was introduced by DESY, [1], as a systematic approach for the quality control of the Niobium used for cavity fabrication.

The basic principle behind this device is to utilize the alteration of the eddy currents, generating a magnetic field in a double coil sensing probe, to detect inclusions and defects embedded under the surface of the material. The Fermilab device [2], Fig. 1, was developed by BAM (Federal Institute for Material research and Testing - Germany) and built by the German company FER-PAused during the SNS cavity fabrication program, and loaned to Fermilab in 2004.

Manuscript received August 29, 2006. This work was supported by the U.S. Department of Energy under contract No. DE-AC02-76CH03000.

Cristian Boffo, author, is with Fermi National Accelerator Laboratory, Batavia, IL 60510 USA (e-mail: crboffo@,fnal.gov).

C. Antoine, C. Cooper and Mike Foley are with Fermi National Accelerator Laboratory, Batavia, IL 60510 USA (e-mail: cantoine@fnal.gov ccooper@fnal.gov foleymh@,fnal.gov ).

Pierre Bauer was with Fermi National Accelerator Laboratory, Batavia, IL 60510 USA. He is now with the EFDA, München, Germany (e-mail: pbauer@efda.org ).

Arne Brinkmann is with DESY, Notkestrasse 85, Hamburg, 22607 Germany (arne.brinkmann@desy.de )

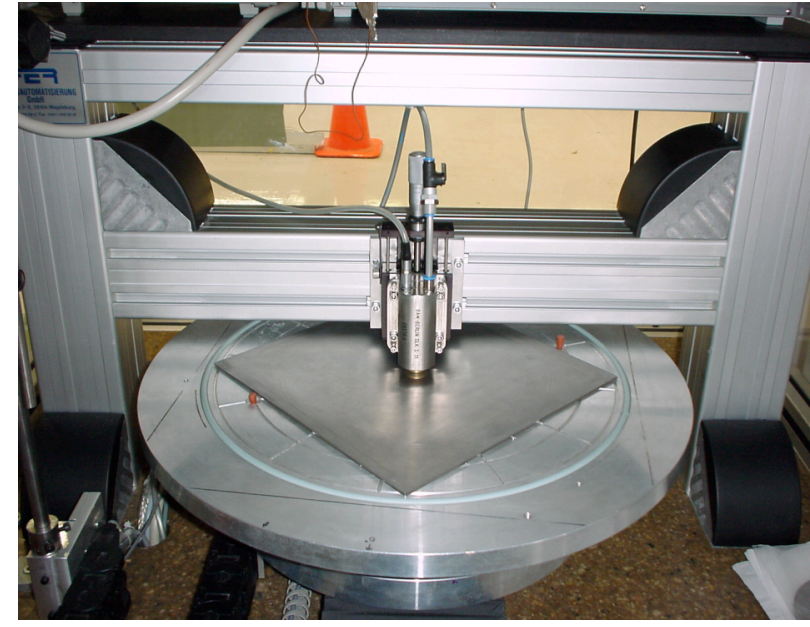

Fig. 1 SNS Eddy current scanner setup

In this particular machine, the sample is fixed to a rotating table, which is attached to a linear slider, while the sensing head is kept fixed. This setup is specifically designed to scan disks: at a given relative radial position of the head, the sample rotates several times allowing for data acquisition, then the relative position of the head is changed by sliding the table and the data acquisition is repeated over a different circumference.

The head generates two signals at different frequencies for simultaneous investigation over two depths. The signals are then digitalized and transferred to a PC where the data is stored and represented in the form of pictures as shown in Fig. 2.

A particular feature of the BAM scanner design is the pneumatic probe levitation. Compressed air is blown through the probe onto the sample, which allows the spring mounted probe to float over the sample. This feature stabilizes the probe to sample distance allowing the probe to instantaneously adapt to irregularities of the sample surface. Since the rotating table uses a vacuum clamping system intended for the SNS blanks, a fixture was designed to adapt the machine to the small niobium disks for the $3.9 \mathrm{GHz}$ cavities developed at Fermilab. When scanning $1.3 \mathrm{GHz}$ squares (10.5"x10.5") the fixture is removed and simple stainless steel or Teflon aligning clamps are used.

As part of the calibration and qualification process, the unit has been benchmarked with respect to the DESY unit initially showing a lower sensitivity that limited the overall detection 


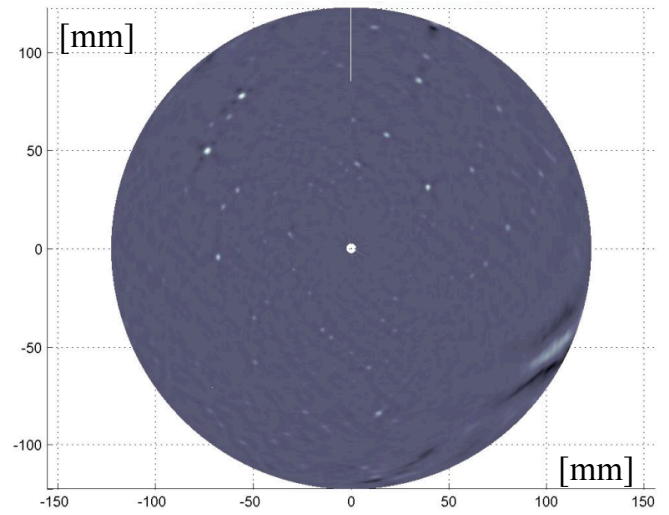

Fig. 2 Scan result of an ILC square after filtering. The white spots correspond to pits on the surface of the material.

capabilities. Now, after several hardware and software upgrades, the device has reached similar performance compared to the DESY one. Since then the Fermilab machine has been utilized to scan over 230 blanks to be used in the fabrication of ILC cavities and several small disks for the third harmonic project.

\section{OPTIMIZATION OF THE SCANNING PROCESS}

The optimal scanning results have been achieved by enhancing both hardware and data analysis aspects of the process [3]. Among others, the most significant improvements were the probe to sample distance reduction and the data filtering implementation.

The distance of the sensing head with respect to the sample is kept stable by the pneumatic probe levitation system. In order to minimize it one must be capable of precisely adjusting the head height and pitch with respect to the sample. For this purpose the head was attached to a micrometric adjusting stage allowing reducing the gap to several $\mu \mathrm{m}$.

At this distance the flatness and the thickness variation of the sample become the limiting factor and cannot be easily overcome.

If the system is properly calibrated, the sample-head distance variations and the inclusions or defects should be separated into the real and in the imaginary portions of the complex number obtained in each scanned point [4]. Often, however, this is not the case. Moreover the distance variations limit the capability of effectively detecting the defects. This problem is related to the way the scanning results are visualized, namely in a picture of the scanned area where the color of each point (usually in gray scale) is proportional to the signal detected. The color range is adjusted to the maximum and minimum values detected. If for example the signal related to a probe-sample distance variation is very strong, the signals related to defects are drowned in the noise and not visible. In order to minimize this effect a new filtering application was developed [5].

Fig. 3 shows the scanned data along a radius with a typical defect (oval) sitting on top of the distance variation effect (dashed line). The new application applies a band-pass finite impulse response filter to eliminate the long-range distance variation effect and therefore resolve more clearly the defect.
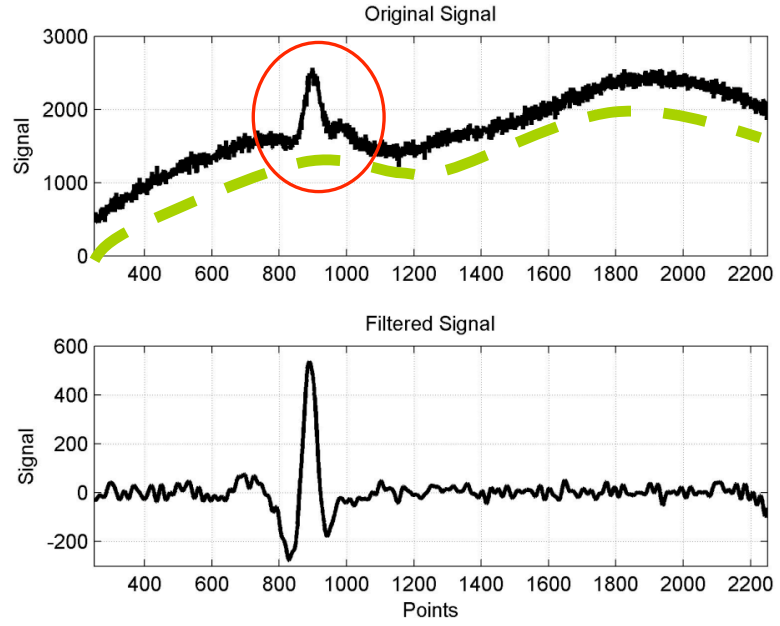

Fig. 3 Data along a radius of a sample with a typical defect. The top plot shows the original signal with the defect (oval) on top of the distance variation effect (dashed line). The bottom plot shows the filtered signal.

\section{ECS RESULTS}

In over two years, more than 400 niobium blanks have been scanned. The blanks can be divided in two categories depending on the type of cavities to be fabricated: third harmonic disks and ILC squares. As far as material for the third harmonic project, a number of 148 disks have been scanned for the fabrication of up to 8 cavities, while 230 ILC squares have been qualified for the fabrication of 12 cavities. In the near future proton driver material for the spoke resonators will be qualified as well. Most of the niobium was purchased from the same supplier and only since lately a second one has been evaluated (small statistic) as part of a single cell $3.9 \mathrm{GHz}$ electropolishing R\&D project. The ECS results are usually summarized in tables showing the preferred side to be used as the RF surface of the cavity. This data however is not considered to be a mandatory selection but just a mere suggestion. A summary of the results for different cavities and suppliers is shown in Table 1.

TABLE 1 EDDY CURRENT SCANNING RESULTS

\begin{tabular}{lcrrrr}
\hline Type & Supplier & Total & OK & $\begin{array}{c}\text { OK 1 } \\
\text { side }\end{array}$ & Reject \\
\hline ILC & A & 230 & 115 & 100 & 15 \\
$3^{\text {rd }}$ harmonic & A & 148 & 57 & 75 & 26 \\
$3^{\text {rd }}$ harmonic & B & 17 & 4 & 11 & 2 \\
\hline
\end{tabular}

\section{ADDITIONAL INVESTIGATIONS}

As part of the quality control process, when defect signatures are detected during the scanning or during the visual inspection, additional techniques are used to further investigate their nature. Several procedures being adopted at FNAL are here discussed.

\section{A. Anodization}

Anodization is the buildup of an oxide layer with welldefined thickness on the material surface conferring to it a characteristic color. In the case of niobium the higher contrast of the colored surface allows to detect inclusions or defects. 


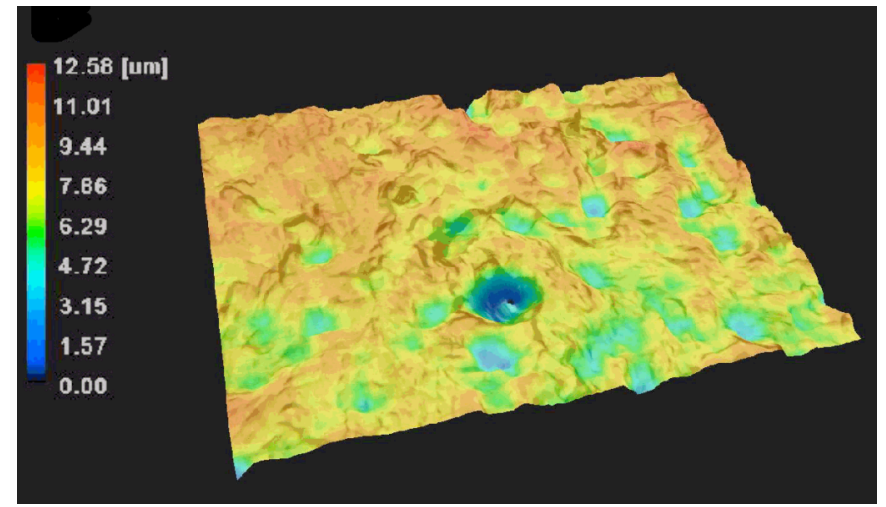

Fig. 4 Small pits detected by the optical microscope but not visible in the ECS results.

By applying 20V to an electrolytic cell composed by $\mathrm{Nb}$ anode and cathode immersed in a 5:1 $\mathrm{H}_{2} \mathrm{O}: \mathrm{HNO}_{3}$. solution, the cathode surface turns into dark blue [6]. As a result of this process, foreign materials with a different surface nature show different color features allowing for a faster detection. No defects were discovered using this technique so far.

\section{B. Profilometry}

In the effort to better understand and characterize the relevance of localized surface defects, several ILC squares were measured with a Taylor Hobsen profilometer. This technique allows detecting the depth of pits and scratches with a precision of few microns. The measurements performed confirm that the scratches are less deep than the pits and will probably be removed during the material processing.

\section{Microscopy}

An extended field of view optical microscope with 3D reconstruction capabilities was used to further characterize the scanner findings. This technique confirmed that the ECS cannot resolve the majority of pits and scratches, which are mostly in the 15-35 $\mu \mathrm{m}$ range, Fig. 4.

Often several surface features of such size have been observed with the microscope in the approximate vicinity of a scanner signal. Being improbable that these "small" defects are its cause, the most likely origin of the scanner signals has been associated to the presence of shallow, large depressions.

Another recurrent feature found on niobium sheets are the so-called "dull spots" (diameter $200 \mu \mathrm{m}$ ). Most likely these are the result of some acidic droplets dried on the surface and creating localized oxidation. Chemical analysis with EDS did not reveal any fluorine or other elements other than those expected ( $\mathrm{Nb}$ and organic contaminants).

\section{Surface FEATURES IDENTIFIED BY THE ECS}

The most common features detected on the surface of the niobium sheets are discussed in this section including pits, scratches, shallow depressions and stripes.

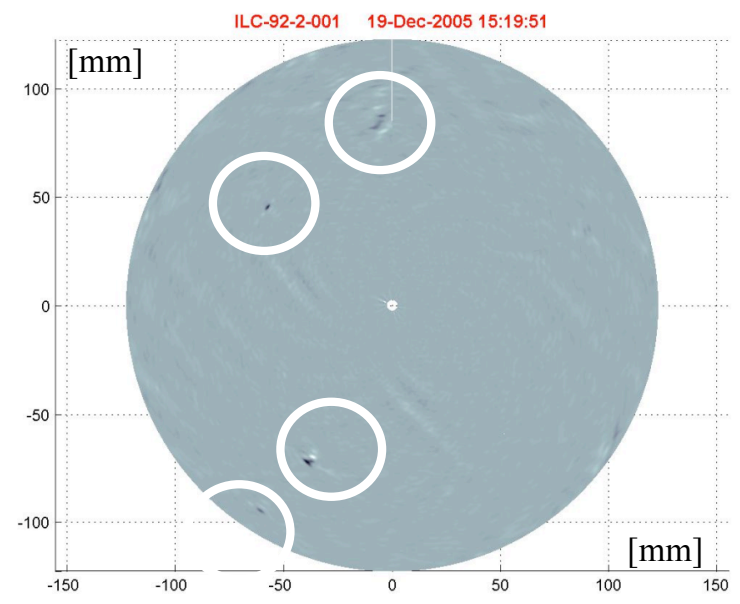

Fig. 5 Typical signatures of shallow depressions on the surface of an ILC square.

\section{A. Pits and Scratches}

When strong signals on a scan result are confirmed by the optical inspection to be deep pits, there are concerns that the material processing will not recover them.

In order to investigate this issue, an ILC square showing heavy pitting was etched six times, $20 \mu \mathrm{m}$ each while the scanning procedure was repeated at each step. As a result it was confirmed that not all the pits had disappeared at the end of the processing. This result is consistent with the observation that the typical chemical process used to remove the niobium superficial layer (buffered chemical polishing, known as BCP) increases the overall surface roughness.

On the other hand, on several other squares, it was confirmed that the superficial conditioning of the material consisting of a light grinding, which is completely removed by a short $20 \mu \mathrm{m}$ chemical etching.

\section{B. Shallow Depressions}

A number of ECS signals have been discovered to be very shallow point like defects $(<10 \mu \mathrm{m})$ much larger in diameter $(>1 \mathrm{~mm})$ with respect to pits. This observation was confirmed by profilometric measurements and 3-D reconstructions produced via optical microscopy. Fig. 5 shows an example of a sheet where 4 different point defects could be identified as shallow and superficially extended depressions. Although not very deep and hardly visible by eye, these defect are detected much easily by the ECS and produce a stronger signature with respect to pits due to the large surface covered. As a matter of fact a pit discovered on an ILC square was measured $\sim 70 \mu \mathrm{m}$ deep and $\sim 70 \mu \mathrm{m}$ in diameter with the profilometer, but appeared to lie at the edge of the scanner resolution. This finding is consistent with the results from the calibration work performed on this scanner [3], which showed that $100 \mu \mathrm{m}$ defects could be resolved in a $100 \mu \mathrm{m}$ depth. Slightly smaller defects can be resolved when closer to or at the surface.

The origin of the shallow depressions could be accidental hits with blunt objects. It has to be noted that high purity niobium is very soft and such imprints happen easily. Unlike deep pits, which we like to avoid, the shallow depressions are probably not impacting RF performance. 


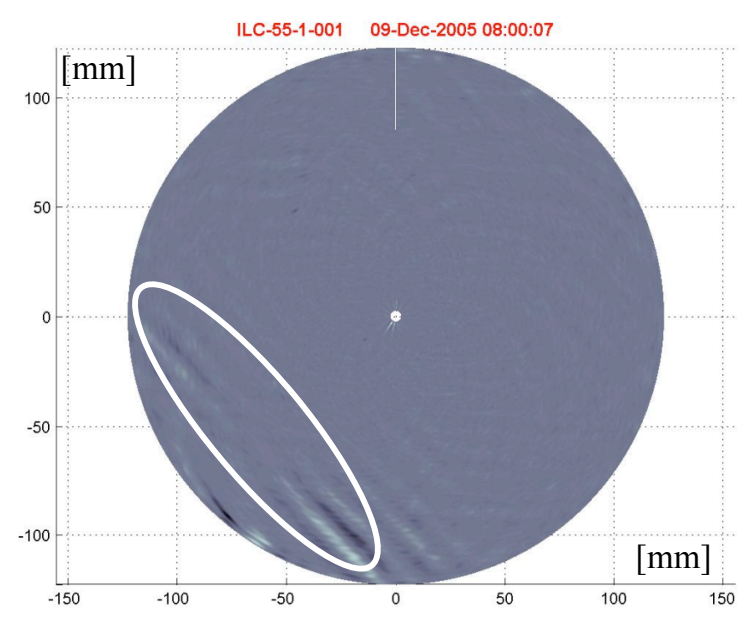

Fig. 6 Typical signatures of stripes on the surface of an ILC square.

\section{Stripes}

The eddy current scanning of the niobium sheets to be used for fabrication of ILC cavities revealed a new feature, "stripes" running parallel to the sheet edges and thus presumably parallel or perpendicular to the rolling direction. These signals are very much like other surface features called "roller marks", except for being strictly parallel to the sheet edges.

These stripes have since been shown to be topological features, i.e. surface waves with peak-to-peak amplitudes of $\sim 4 \mu \mathrm{m}$ and spaced by a little less than $25 \mu \mathrm{m}$, Fig. 6 . Profilometric stylus measurements across and along these features on several squares confirmed the scanner results indicating long range variations of even larger amplitude in both directions. In addition a fast fourier transform analysis of the data acquired by the profilometer confirmed the periodicity of the waves detected by the scanner.

Additional support for the explanation of the stripes in the ECS results as surface topology came from an etching test. Several sheets were etched for 10 minutes using standard BCP and scanned again showing that the stripes partially disappeared after removing a $10 \mu \mathrm{m}$ surface layer. During the same test, the squares also underwent a high temperature heat treatment (in vacuum), which did not affect the stripes signals in the ECS.

Unfortunately, these results could not be confirmed independently by optical interferometry, but since all these height variations do not violate the specification for the material, they are not expected to affect the cavity performance. The most likely explanation of this surface feature is that the stripes could be an artifact of small imperfections in the rolling process (e.g. play in the rollers, bending of sheets while clamped between the rollers, etc.).

Lately the sensing probe has been reworked and its calibration is in progress while the ultrasonic technique is being evaluated as a possible alternative or complementary process to ECS.

\section{CONCLUSION}

Eddy current scanning as a quality control process of niobium to be used for SRF cavity fabrication is now being routinely applied at FNAL for two years. Additional techniques such as anodization, profilometry and microscopy have been implemented to support the ECS results and to further investigate the findings.

The majority of the detected signatures have been correlated to surface topological defects while only two candidates have been classified as possible inclusions. Most of the findings are scratches, pits, shallow depressions and stripes.

\section{ACKNOWLEDGMENT}

The authors are very thankful to Dan Stout and Norbert Holtkamp from SNS for the transfer of the SNS-ECS to Fermilab and to Helen Edwards and Harry Carter for the support of this activity. We would also like to thank Ralph Casperson from BAM for his advice. We are most grateful, however, to Marco Battistoni, Oscar Lira and Robert Schuessler who conducted most of the ECS measurements presented here.

\section{REFERENCES}

[1] W. Singer, D. Proch, A. Brinkmann, "Diagnostic of Defect in High Purity Niobium", Proceedings of the RF Superconductivity workshop VIII, Abano Terme, Italy, Oct. 1997.

[2] P. Bauer et al., "Eddy-Current Scanner Operating Instructions", Fermilab, Technical division, internal note TD-04-029, Jun. 2004.

[3] C. Boffo et al., "Eddy Current Scanning at Fermilab", Proceedings of the RF Superconductivity workshop 2005, Cornell University, Ithaca NY USA Jul. 2005.

[4] D. J. Hagemaier, "Fundamentals of eddy current testing", The American Society For Nondestructive Testing, Feb. 1990.

[5] C. Boffo et al., "FIONDA", Fermilab, Technical Memo, internal note TM-2313-TD, Jun. 2005.

[6] D. Proch, "DESY Specification of the welded $1.3 \mathrm{GHz}$ SRF resonators for the TTF-FEL", DESY technical document, 1999. 